Cuadernos de Filología Clásica. Estudios Latinos

ISSN: 1131-9062

http://dx.doi.org/10.5209/CFCL.56190

\title{
Un nuevo manual de enseñanza: los Rhetoricae artis progymnasmata de Thomas Blebel ${ }^{1}$
}

\author{
$\mathrm{M}^{\mathrm{a}}$ Elena Curbelo Tavíoº
}

Recibido: 10 de abril de 2016 / Aceptado: 25 de febrero de 2017

Resumen. El humanista alemán Thomas Blebel publicó en Leipzig, en 1582, un tratado titulado Rhetoricae artis progymnasmata: Ex optimis quibusque autoribus methodice conscripta, exemplisque tam sacris, quam philosophicis illustrata, \& ad puerilem institutionem accommodata, que pretendía ser, como adelanta desde el título, un compendio de ejercicios retóricos preparado y dirigido a estudiantes. El objetivo de este artículo es la descripción y el análisis de su manual con el fin de demostrar que, pese a su título inicial, la obra de Blebel poco tiene que ver con los manuales de progymnasmata tan en boga en su época

Palabras clave: retórica; humanismo; progymnasmata; Blebel.

\section{[en] A new teaching manual: Rhetoricae artis progymnasmata by Thomas Blebel}

\begin{abstract}
The German humanist Thomas Blebel published Rhetoricae artis progymnasmata: Ex optimis quibusque autoribus methodice conscripta, exemplisque tam sacris, quam philosophicis illustrata, $\&$ ad puerilem institutionem accommodata in Leipzig, 1582. As the title reads, the work was devised as a compendium of rhetorical exercises targeted at and prepared for language learners. This paper presents a description and analysis of Blebel's manual to demonstrate that, despite the original title, this work does not align with the progymnasmata manuals so popular at the time.
\end{abstract}

Keywords: Rhethoric; Humanism; progymnasmata; Blebel.

Cómo citar: Curbelo Tavío, ME., «Un nuevo manual de enseñanza: los Rhetoricae artis progymnasmata de Thomas Blebel, Cuad. Filol. Clásc. Estud. Lat. 37.1 (2017), 119-28.

El pedagogo y rector de la Universidad bávara de Hof, Thomas Blebelius (Thomas Blebel, Thomas Blebel d. Ä. [der Ältere]) nació en 1539 en la ciudad alemana de Bautzen, capital de la Alta Lusacia. Durante su periodo de formación, coincidió en Wittenberg con el filósofo y teólogo Johannes Sturio (1525-1562) y tuvo entre sus preceptores al humanista Philipp Melanchthon (1497-1560), al médico y matemático

\footnotetext{
1 Este trabajo se ha realizado en el marco del Proyecto de Investigación FFI2014-53859-P La pervivencia de los progymnasmata de Aftonio y Teón en las preceptivas retóricas del Humanismo: reescritura y manipulación de los ejercicios de primera enseñanza, financiado por el MINECO.

2 Universidad de Las Palmas de Gran Canaria. Instituto Universitario de Análisis y Aplicaciones Textuales.

E-mail: mariaelena.curbelotavio@ulpgc.es
} 
Bartholomeus Shönborn (1530-1585) y a su convecino el reformador Caspar Peucer (1525-1602), por cuyo consejo se trasladó a la Universidad de Hof, en la que desempeñó los cargos de maestro de quartus (1561), preceptor (1573), vicerrector (1575) bajo el mandato del pedagogo luterano Christoph Cadesreuter (-1589) y, finalmente, el de rector en 1591. Tras 35 años dedicados a la enseñanza, murió el 25 de marzo de 1596 a la edad de 57 años. ${ }^{3}$

$\mathrm{Su}$ importante labor pedagógica se plasma en sus tres principales obras, todas ellas destinadas al ámbito docente y adaptadas a los escolares a los que, en último término, iban dirigidas: De Sphaera et primis Astronomiae rudimentis Libellus ad usum scholarum maxime accomodatus (Wittenberg, 1578); Rhetoricae artis progymnasmata: Ex optimis quibusque autoribus methodice conscripta, exemplisque tam sacris, quam philosophicis illustrata, \& ad puerilem institutionem accommodata (Leipzig, 1582); Grammatices Hebraeae sanctae linguae institutiones (Wittenberg, 1587), reeditada y adecuada esta última por su hijo para el uso de los estudiantes bajo el título Compendium Hebraeae linguae, ex Grammatica Blebelii Parentis, quanta fieri potuit breuitate \& perspicuitate, collectum, \& ad puerilem institutionem omnino accomodatum (Wittenberg, 1594).

Como se ha mencionado, Blebel compuso en Leipzig, en 1582, un tratado titulado Rhetoricae artis progymnasmata: Ex optimis quibusque autoribus methodice conscripta, exemplisque tam sacris, quam philosophicis illustrata, \& ad puerilem institutionem accommodata.

Este manual pretendía ser, como adelanta desde el título, un compendio de ejercicios retóricos preparado y dirigido a estudiantes del primus ordo por encargo del Superintendente e Inspector de su universidad, Aurelius Streitberger (1543- 1612). Sin embargo, su obra no es sino otro tratado de retórica, muy pedagógico en su forma, pero muy similar a los que circulaban en su época y alejado de los manuales de progymnasmata 4 que transitaron a lo largo del humanismo.

La edición consultada es la impresa en octavo en Leipzig en 1584 por Johannes Steinman $(-1588)^{5}$, editor también de la obra de Melanchthon entre otros y vendida por el librero y editor Henning Gross (1553 - 1621). Se compone de 608 páginas, de las que el texto de Blebel ocupa 303 organizadas en 19 cuadernillos. El resto se corresponde a las hojas de guarda así como a la inclusión, dentro del cuerpo del texto, de una serie de páginas en blanco, que se presentan regularmente cada dos hojas del manual, y a la adición, al inicio del texto (justo antes del título) y después de la carta dedicatoria, de algunas páginas manuscritas sobre las partes del discurso ( $D e$ inuentione; De dispositione).

La mano que redacta estas páginas parece ser la misma que, a la par que al texto editado, realiza anotaciones y correcciones marginales y supralineales tanto en la carta introductoria como en los capítulos iniciales. De este modo, junto al término griego Progymnasmata del título, encontramos manuscrita su traducción latina, praeexercitamenta, así como otra serie de aclaraciones, también a mano, difíciles de leer. Similares

Para una biografía más detallada del autor puede consultarse el capítulo que Gottfried Ludovici (1708, 283-289) le dedica en el primer volumen de su obra Historia rectorum, gymnasiorum scholarumque celebriorum.

4 Sobre progymnasmata y su evolución desde su posición en el mundo clásico hasta el siglo XXI, véase Arcos Pereira (2015).

5 CERL Thesaurus: accessing the record of Europe's book heritage: < http://thesaurus.cerl.org/record/ cnp01333437> [16/02/2016]. 
anotaciones están repartidas a lo largo de todo el texto inicial (concretamente hasta la página 22 del manual), tratándose algunas veces de meras traducciones o aclaraciones de palabras o expresiones griegas que se considera necesario glosar, como el término griego Erotemata, sobre el que sitúa el latino quaestiones o el sintagma praeceptionum ubertatem, aclarado igualmente en línea superior con la expresión copiam praeceptionum, ambos en la nota admonitoria con la que comienza el manual.

Los 19 cuadernillos que componen esta edición tienen todos 8 folios. En el margen superior del manual, propiamente dicho, se encuentra, tanto en el folio recto como en el verso, la paginación con numeración arábiga ${ }^{6}$ y el título o subtítulo que permite identificar el contenido del texto de la hoja. El margen inferior presenta en el borde derecho, también por ambos lados, como reclamo, la primera sílaba de la palabra inicial del folio siguiente para facilitar su encuadernación. La parte central del margen inferior de los folios rectos ofrece igualmente la identificación del cuadernillo con una letra mayúscula y la numeración.

El libro se inicia con el título de la obra, el nombre del autor y la fecha y lugar de edición. Termina con el colofón Finis Rhetoricae. Lipsiae imprimebat Ioannes Steinman anno MDLXXXIIII impensis Henningi Grossii Bibliop. Tras este se ha añadido una nota que advierte sobre la presencia de una sinopsis bajo la forma de un esquema recopilatorio o tabula que, sin embargo, no se incluye en la edición: Sequitur Synopsis omnium, quae in hoc toto libro de quinque Rhetoricae partibus tractantur in breui tabula adumbrata. $(\mathrm{p} .302)^{7}$

Por último, se encuentra una carta de elogio y aprobación de la obra de Blebel del humanista y pedagogo alemán Johann Rivius Atthendoriensis, "el Joven" (15281596), dirigida al rector de la Universidad de Leipzig, Joannes Cramerus (Johann Cramer). En ella se elogia la editio huius opusuculi Blebeliani tanto por la cantidad y variedad de preceptos, ausentes en otros manuales, como por la selección de los ejemplos: Primum propter plurima specialia praecepta, quae in reliquis, qui circumferuntur libellis, non exstant. Deinde propter delectum exemplorum, et illorum satis eruditam oeconomiam et dispositionem, in sacris, et prophanis ostensam. (p.303)

Previa a la carta dedicatoria, el tratado comienza con un aviso al lector (Admonitio ad lectorem), que no es más que una recomendación para aquellos profesores que, debido a la abundancia de preceptos que Blebel proporciona en su manual, no lo consideran apto para estudiantes. En tal caso deja abierta la posibilidad de que cada cual, según su juicio y previsión, adapte y elija de su obra aquello que sea adecuado a las necesidades personales.

Si qui iudicabunt haec Rhetoricae Erotemata, propter praeceptionum ubertatem ad puerilem institutionem non satis accommodata, poterunt pro suo iudicio et prouidentia ad captum puerorum sese demittentes inde solummodo ea eligere, et proponere, quae ingeniis et uiribus discentium conuenientia et maxime necessaria uidebuntur, ex quorum numero sunt, quae hoc signo: $\uparrow$ enotari curauimus, quibus probe cognitis ad reliqua perdiscenda, uia erit plana et facilis (p.2)

$6 \quad$ Hay un error en la paginación pues se repite el número 63 en dos páginas diferentes. A partir de ahí, las páginas pares están en el folio recto, las impares, en el verso.

7 Todas las referencias que se ofrecen de la Rhetoricae artis progymnasmata de T. Blebel se citan por el número de página que presenta la edición de Leipzig de 1584 y no con el número de folio. 
La carta inicial está dedicada a los más importantes y prudentes decuriones, cónsules y senadores de la ciudad germana de Amberg, a los que elogia con gran número de epítetos. Esta carta constituye una introducción a la obra en la que el maestro bávaro presenta, por un lado, las características imprescindibles en todo buen profesor, la fidelitas y la honestitas. Por otro lado explica también el estilo que va a seguir en la exposición, esto es, el método de pregunta-respuesta, por considerar que ello facilita el aprendizaje.

Al utilizar este método de preguntas directas con respuesta corta, Blebel entronca tanto con el modelo pedagógico de los catecismos protestantes, como con la tradición clásica, pues este método se encuentra en las Partitiones Oratoriae de Cicerón y en los Rhetores Latini Minores. Como afirma Rodríguez Herrera (2014, 268), esta metodología, de corte escolástico, no es anacrónica, ya que responde a los presupuestos pedagógicos buscados por ciertos humanistas en sus obras. Así lo vemos, por ejemplo, en dos autores contemporáneos de Blebel, como son el teólogo luterano Burchardus Harbart y el matemático y humanista alemán Georg Henisch Bartfeldensis, quienes se sirven de este formato en sus In Aphthonii Sophistae Progymnasmata Commentatio y en los Praeceptionum rhetoricarum libri V et exercitationum libri II, respectivamente (Rodríguez Herrera 2016, 176).

Merito igitur illam cum aliis peritis scholarum gubernatoribus docendi rationem sequimur, quae antiquissima habetur, et multorum iudicio utilis studiis puerilibus uidetur, atque Erotematis seu interrogationibus et responsionibus accommodatis instituitur, et ad artes integre discendas facilius acceditur. (p.4)

De igual modo ofrece las fuentes de su enseñanza, entre las que destacan algunos de sus preceptores y compañeros en Wittenberg, los reformadores Philipp Melanchthon (1497-1560), Nikolaus Medler (1502-1551) y Lucas Lotze (1508-1582), además de los discursos de Cicerón:

Ego quoque, qui nunc, Dei beneficio, uiginti tres annos in hac Curiana schola publico docendi munere cum collegis dilectis et uiris reuerendis fungor, inter alias operas ac maiora exercitia scholastica discipulis nostris primi ordinis Rhetoricae artis Erotemata, ex Domini praeceptoris Philippi Melachthonis praeceptis, pro rudioribus, et clarissimis, et reuerendis uiris D. D. Medlero et L. Lossio de Ecclesia Christi et scholis optime meritis collecta, hactenus proposuerim, eorumque usum ex integris orationibus Ciceronis et aliorum autorum scriptis declarauerim. (p.5)

Pero, sobre todo, esta carta es una declaración de intenciones en la que no faltan las razones que lo llevan a la redacción de su manual y su propósito al reunir los preceptos retóricos necesarios para un correcto dominio de la oratoria, cuyo conocimiento se muestra imprescindible tanto para los hombres de Iglesia como para los de Estado, en el ámbito público y en el privado. La utilidad del estudio de la elocuencia viene ilustrada con varios ejemplos, algunos extraídos de los discursos del propio Melanchthon (1541, p.142).

La epistola dedicatoria, datada en Curia Variscorum, actual Hof, el 25 de diciembre del año 1584, finaliza con el agradecimiento a los miembros de la Escuela de Amberg a la que Blebel pertenece. La parte introductoria concluye con un Epigramma que el jurista alemán y también estudiante de la Universidad de Wittemberg 
Johannes Albinus (1540-1602) dedica al autor de Bautzen y que define la obra como Artis opus.

El manual, propiamente dicho, se inicia con unas cuestiones generales de retórica o prolegomena a las que da el título de Quaestiones rhetoricae ad puerilem institutionem accomodatae atque utilibus exemplis illustratae. Aunque las definiciones de rhetorica y dialectica son las clásicas, las palabras de Blebel están prácticamente tomadas de Melanchthon y de Lotze:

Eloquentia facultas est sapienter et ornate dicendi [...] Rhetorica uero est ars, quae docet uiam ac rationem recte et ornate dicendi. [...] Vero hoc interesse dicunt quod Dialectica res nudas proponit, Rhetorica uero addit elocutionem quasi uestitum. (Melanchthon 1537, $\mathrm{A}^{\mathrm{v}}$ y $\mathrm{A} 6^{\mathrm{rv}}$ )

Dialectica est ars seu uia, recte, ordine et perspicue docendi [...] Dialectica circa omnes materias uersatur, et rerum summas uerbis nudis proponis, sed rethorica addit ornatum iis materiis quae orationis copia et splendore illustrati et uarie depingi possunt. (Lossius 1600, $\mathrm{B} 1^{\mathrm{rv}}$ )

\section{Quid est Rhetorica?}

Rhetorica est ars, quae docet uiam ac rationem recte et ornate dicendi, quae facultas Eloquentia uocatur. [...]

Quid differt a Dialectica?

Etsi Rhetorica cognata est dialecticae, eique quasi ex altera parte, ut ait Aristoteles, respondet, differunt tamen inter se et forma et fine.

Quomodo forma?

Forma siue modo tractandi differunt, quod Dialectica nude et simpliciter sine uerborum ambagibus de rebus disserit. Rhetorica uero addit elocutionem quasi uestitum. (Blebel pp.19-20)

A ellas añade, siguiendo siempre el mismo método pedagógico de preguntarespuesta, la definición de las distintas partes de la retórica, para las que elige la terminología de QVINT.Inst.3.38: Quot sunt partes Rhetoricae? Quinque: Inuentio, Dispositio, Elocutio, Memoria et Pronunciatio. (p.20)

A continuación, el texto se presenta como un manual de retórica al uso muy completo y muy didáctico. No hay que olvidar que el propio Blebel, ya desde el título, anuncia su obra como ad puerilem institutionem accommodata. Esta adaptación al ámbito escolar se observa en la estructura del texto, muy organizado, en el que cada capítulo y apartado están perfectamente identificados, numerados, ejemplificados e, incluso a menudo, resumidos a través de un esquema o tabula ${ }^{9}$.

El manual se divide en cinco partes, tantas como tiene la retórica clásica, y cada una de estas se presenta y concluye con su título y el lugar que ocupa en la preceptiva. De este modo encontramos un largo capítulo dedicado a la inuentio, De inuentione. Prima Rhetoricae parte; seguido de dos algo más cortos, De dispositione.

8 Omnis autem orandi ratio, ut plurimi maximique auctores tradiderunt, quinque partibus constat: inuentione, dispositione, elocutione, memoria, pronuntiatione siue actione (utroque enim modo dicitur).

9 A esta misma adaptación somete su hijo T. Blebel, como ya se mencionó anteriormente, el compendio de lengua hebrea realizado a partir de la obra de su padre Grammaticae Hebraeae sanctae linguae institutiones (1587) (Ludovici, 1708, 284). 
Secunda Rhetoricae parte y De elocutione. Tertia Rhetoricae parte respectivamente; y, por último, otros dos capítulos muy breves centrados en las dos últimas partes de la retórica, De memoria. Quarta Rhetoricae parte y De pronunciatione. V et ultima Rhetoricae parte.

El primer capítulo, De inuentione. Prima Rhetoricae parte, nos sirve de modelo para exponer la estructura del manual, que se mantiene a lo largo de toda la preceptiva: en primer lugar, la definición y características generales de la parte de la retórica que está tratando; continúa con una explicación pormenorizada de todos los elementos y las características particulares de esta parte, en un orden estricto, pues marca cada apartado y subapartado bien con un título o epígrafe, bien con diferente tamaño de letra, o bien con números; esta detallada explicación se ofrece siempre bajo la forma pedagógica de pregunta-respuesta: Quare inuenta sunt genera causarum? Propter duas causas. I. Vt suppeditent locos ad tractandam materiam propositam. [...] II. Deinde ut monstrent statum unius cuiusque scripti. [...] (p.24)

En este primer capítulo, tras la definición de inuentio ${ }^{10}$, se añade la de cada uno de los genera causarum. Blebel define los géneros retóricos, añadiendo a los ya clásicos deliberativo, demostrativo y judicial, el didascálico, en lo que sigue, por tanto, la doctrina de Melanchthon:

Vulgo tria numerant genera causarum. Demonstratiuum, quo continetur laus et uituperatio. Deliberatiuum, quod uersatur in suadendo et dissuadendo. Iudiciale quod tractat controuersias forenses. Ego addendum censeo $\delta 1 \delta \alpha \sigma \kappa \alpha \lambda \iota \kappa o ̀ \mu$ (sic) quod etsi Dialecticam pertinet [...] non est praetermittendum, praesertim cum hoc tempore uel maximum usum in Ecclesiis habeat. (Melanchton, 1537, A7 rv ).

Sin embargo, al contrario que él, el autor de Bautzen lo sitúa en primera posición:

Quot sunt genera causarum?

Pro quadruplici quaestionum forma, quatuor genera causarum constituuntur, $[\ldots]$ Quae sunt illa?
I. Didascalicum,
III. Deliberatiuum,
II. Demonstratiuum,
IIII. Iudiciale. (p.22)

Antes de explicar ampliamente las partes del discurso y la teoría de los genera causarum, el maestro bávaro define la stasis y ofrece su etimología en un breve apartado denominado De statibus. Un ejemplo bíblico, el Salmo 9, y uno clásico, el discurso de Cicerón Pro Marcello, le sirven para ilustrar la teoría de la stasis y de los géneros retóricos, así como para concluir el apartado. Blebel tratará la stasis, con posterioridad, dentro de cada uno de los genera causarum, especialmente en el capítulo dedicado al genus iudiciali.

De partibus orationibus comprende el análisis de cada una de las seis partes del discurso bajo los epígrafes De exordio, De narratione, De propositione, De confirmatione, De confutatione y De peroratione. Incluye, dentro de cada una de las partes, su definición, sus características y los tipos o species que se pueden

10 Quare Inuentio primo loco ponitur? Quia prius reperire debet Orator rei, de qua dicturus est, naturam, uim, partes etc. quam disponat, aut oratione ornet. (p.21) 
encontrar en ellos, la etimología griega cuando es posible y numerosos ejemplos clarificadores.

A continuación, se expone la teoría de los géneros retóricos, dentro de cada uno de los cuales se definen y ejemplifican igualmente cada uno de los subgéneros. En este caso, además del título, aparece una numeración: De didascalico, primo causarum genere; De II causarum genere. De genere demonstratiuo; De deliberatiuo. Tertio Causarum genere y, por último, De iudiciali. Quarto causarum genere.

En el género demostrativo, enumera igualmente las tres species o clases en las que se divide este género: De I classe materiarum. De laude personarum; II Classis materiarum, generis demostratiui. Laudatio factorum; III Classis materiarum, generis demostratiui. Laudatio rerum. Esta última clase, a su vez, se subdivide en otras tres ordines, de nuevo perfectamente organizadas y numeradas: De I ordine. De laudatione rerum incorporearum; De II ordine laudationis rerum corporearum et quidem animantium; De III ordine laudationis rerum: nempe locorum.

Todo lo expuesto muestra cómo Blebel adapta la ardua teoría retórica al ámbito didáctico y al público juvenil al que iba dirigido.

La misma estructura de este amplio capítulo dedicado a la primera parte de la retórica y que acaba con un Finis primae partis rhetoricae de inuentione, se repite a lo largo de los cuatro siguientes en los que minuciosamente se desgrana la teoría de la dispositio, apartado también muy extenso, pues sitúa cada una de las partes en las que se divide la disposición (exordium, narratio, propositio, confirmatio, confutatio y peroratio) en cada uno de los géneros retóricos y lo resume a continuación con una tabula o esquema; la de la elocutio, capítulo en el que se incluyen un tratado sobre puntuación y otro sobre figuras titulados, respectivamente, De compositione y De figuris; la teoría referida a la memoria y, finalmente, un quinto y último, como afirma el propio Blebel, sobre la pronuntiatio con el que pone fin a su retórica (finis rhetoricae).

Un análisis más profundo merece el repertorio de ejemplos que, en gran número, ilustran el manual de Blebel y que, como ya se advirtió al inicio, lo hizo merecedor del elogio de sus contemporáneos.

Los ejemplos se muestran integrados a menudo en la definición y entre ellos alterna los bíblicos, extraídos fundamentalmente de los Salmos y de diversas Cartas de los apóstoles ${ }^{11}$, con los clásicos griegos y latinos. Encontramos el empleo de términos religiosos y referencias bíblicas pertenecientes tanto al Antiguo como al Nuevo Testamento (Génesis, Deuteronomio, Libro de Job, Libro de la Sabiduría, Evangelios de San Mateo, San Juan y San Lucas, Epístolas a los Romanos y a los Corintios, Hechos de los Apóstoles...) sobre todo en el desarrollo del género didascálico, estrechamente unido a la retórica de la predicación eclesiástica. Véase el siguiente ejemplo: Sacra scriptura diserte Spitirum Sanctum uocat Deum et Iehouah, quod solius Dei proprium nomen est, 2. Reg. 22, Esa.6, Actor 6 et 2 Cor. 3, I Corinth. 6, etc. Ergo Spiritus Sanctus est uere Deus. (p.77).

De entre los ejemplos clásicos sobresale el uso de los discursos ciceronianos, como él mismo advirtió que haría en la carta dedicatoria, pues cita a menudo el Pro

11 Habetne quaelibet oratio seu scriptum unicum tantum statum et causae genus? Minime. [...] Da exemplum? Psalmus 9 est generis deliberatiui (p.25); [Confirmatio] Quae causae? Causa efficiens temperantiae est Spiritus Sanctus accedens in mente cogitationem uerbi diuini, quod praecipit temperantiam, Lucae 21 [...] Eph. 5 et Rom. 13 (p.72) 
S. Roscio, el Pro Milone o el Pro Archia, entre otros. En número menor incluye ejemplos de las Geórgicas y de la Eneida de Virgilio, de las Metamorfosis de Ovidio y de la Institutio oratoria de Quintiliano, que es utilizado, junto con Aristóteles, como fuente de algunos de sus preceptos ${ }^{12}$. En alguna ocasión aparece también citado, a modo de ejemplo, Melanchthon ${ }^{13}$ : [De laudatione] Da exemplum? Illustria exempla integrae laudationis personarum petantur ex Plutarcho et aliis: Item ex tomis declamationum Philippi Melanchthonis (p.83)

Blebel ofrece generalmente las fuentes de donde extrae los ejemplos. En muchas ocasiones lo reproduce completo:

Vt Ouidius Metamorphose $\tilde{v}$ ( sic) libros hoc modo orditur: In noua fert animus mutatas dicere formas corpora, dii coeptis, nam uos mutastis et illas, aspirate meis, primaque, ab origine mundi, ad mea perpetuum deducite tempora carmen. Sic Virgilius a propositione sui operis orditur: Arma uirumque cano. (p.33)

Otras veces se limita simplemente a la cita (Quomodo Cicero eam definiat uide, lib. 1, officiorum $)^{14}$, al nombre del autor que se puede consultar como modelo (Exempla in lectione Ciceronis et aliorum ubique obuia sunt. [p.37]) o bien acorta el ejemplo (Esaiae 45. Non obliuiscar tui, etc. [p.143]) o lo abrevia eliminando palabras (Et 2 Timoth 3 omnes qui pie uolunt uiuere, persecutionem patientur [p.142] ${ }^{15}$; incluso, lo parafrasea adaptándolo según sus necesidades: His saltem meminerint iuniores regulam, quam Quintilianus de insinuatione preaescribit, ut uidelicet ab iis, quae laedunt, ad ea quae prosint confugiamus, et si causa laboramus, persona subueniat uel e contra (p.29) ${ }^{16}$.

Resulta llamativo que, pese a que tanto en el título de su obra como en la carta inicial alude directamente a los progymnasmata, la única referencia directa a ellos dentro del manual la encontramos en un ejemplo. El apartado dedicado a la Vituperatio personarum dentro del género demostrativo es ilustrado por Blebel con un ejemplo a imitación de Aftonio: Integrum exemplum uituperationis Paridis ad imitationem Aphtonii (p.84).

Blebel no tiene en su manual un apartado dedicado a los progymnasmata ${ }^{17}$. Solo encontramos alguna mención como acabamos de ver en el ejemplo empleado en la uituperatio ad personam o ligeras coincidencias. Puede observarse un ejemplo de ello en el apartado del tratado dedicado a la Narratio, en el que se aprecia cierta similitud entre Blebel y Aftonio en la exposición que ambos ofrecen de las virtudes y los elementos del relato:

12 Vnde sumitur confutatio? [...] Siquidem omnis res, ut inquit Aristoteles, per quascumque causas nascitur, per easdem dissoluitur. (p.57)

13 Da exemplum laudationis regionum [...] Plura exempla petantur a Cosmographis, Historiographis, Salustio, Iustino, Homero, Virgilio, Ouidio et aliis in primis uero est Declimationum [sic] Tomis D. Philippi (pp.108-109)

14 Quomodo autem in his exordia tractari conueniat docet Cicero libro primo de Inuentione. (p.29); Prudentia oratoris est summam rei de qua dicturus est [...] Quintil. Lib. 4. Cap. 5 (p.48); Confirmatio. Quid nominis? Temperantia eadem [...] id est, seruatrix sapientiae teste Aristotele, lib. 6, Eth. Cap.5. (p.71)

15 La cita completa es la siguiente: omnes etiam qui pie uiuere uolunt in Christo uolunt uiuere, persecutionem patientur (IITim.3.12)

16 La cita exacta (QVINT.Inst.4.1.44) es: Illud in uniuersum praeceperim, ut ab iis quae laedunt ad ea quae prosunt refugiamus: si causa laborabimus, persona subueniat, si persona, causa.

17 Un estudio sobre la presencia de progymnasmata en Blebel aparecerá próximamente en Ágora: estudos clásicos em debate. 
Quae sunt uirtutes seu conditiones Narrationis? Quattuor: Breuitas, Perspicuitas, Probabilitas et suauitas. [...]

Quae est suauitas narrationis? Suauitas siue iucunditas est, quae affectibus, uerbis et gestibus conuenientibus [...]

Quibus rebus constat omnis Narratio? Locis circumstantiarum, qua accidentia narrationis dicitur.

Vnde petuntur? A personis, rebus gestis, causis factorum, consiliis, loco, tempore, modo et euentu, quae in uulgari uersiculo continentur: quis, quid, ubi, quibus auxiliis, cur, quomodo, quando? (pp.40-41)

Narrationes accidunt sex: persona faciens, res gesta, tempus circa quod, locus in quo transacta, modus quo pacto, causa propter quam. Virtutes eius quattuor: Claritas seu perspicuitas, breuitas, probabiblitas, qua $\pi \imath \theta \alpha$ vó $\eta \varsigma$ dicitur, electorum uerborum proprietas. (Lorich 1598, p.23-24)

Como se ha podido ver, Rhetoricae artis progymnasmata es un tratado de retórica, muy pedagógico en su forma, pues ofrece preceptos claros y ejemplos de sus enseñanzas, pero muy similar a los que circulaban en su época. Entre ellos cabe citar los de sus preceptores Melanchthon y Lotze.

Las coincidencias entre el tratado de Blebel y los Erotemata Dialecticae et Rhetoricae Philippi Melanchthonis et Praeceptionum Erasmi Roterodami de Lucas Lotze, dejando aparte la definición de dialectica que se comentó anteriormente, parecen limitarse a la estructura y forma pedagógica que ambas presentan, pues el manual de Lotze ofrece su preceptiva, al igual que el blebeliano, bajo la forma de preguntarespuesta e incluye esquemas, tablas recopilatorias, división numérica, etc.

En las enseñanzas que Philipp Melanchthon ofrece en sus Elementorum rhetorices libri duo sí se observa la fuente de algunos de los preceptos que recoge Blebel en su manual. Las coincidencias se encuentran, fundamentalmente, en el primero de los dos libros que componen la retórica del reformador alemán. Algunos de estos preceptos comunes se han ido presentando a lo largo de este artículo, tales como la definición de retórica, la teoría sobre la distinción entre dialectica y rhetorica o la adición del género didascálico a la nómina de los genera causarum.

El orden en el que se presentan los preceptos en el primer libro de Melanchthon y en el manual de Blebel es prácticamente el mismo, si bien difieren en la disposición de los géneros retóricos. Por otro lado, aunque ambos están dirigidos a la enseñanza de estudiantes, pues el tratado del teólogo alemán está dedicado a Albrecht (c. 1575) y Johann W. Reiffenstein (c. 1520-1575), hijos de su amigo el humanista Wilhem Reiffenstein (1482-1538), y el del pedagogo de Bautzen a los estudiantes del primus ordo por encargo del director de su universidad, es la de este último una preceptiva mucho más didáctica. A este hecho ayuda un uso mucho menor del griego, una presencia muchísimo mayor de ejemplos y una estructura mucho más clara con definiciones breves y concisas, a las que, sin lugar a dudas, contribuye el método catequético de pregunta-respuesta del que se sirve durante todo su tratado.

Podemos concluir que la obra de Blebel es una adaptación muy organizada y didáctica ad puerilem institutionem accomodata de los manuales de retórica humanistas, a lo que ayuda el gran número de ejemplos y un menor uso del griego, alejada de los tratados de progymnasmata que transitaron a lo largo del humanismo para la enseñanza de los primeros niveles de aprendizaje de esta disciplina. 


\section{Referencias bibliográficas}

Arcos Pereira, T. (2015), «Los primeros niveles de la enseñanza de la retórica en Europa: los progymnasmata», en Maestre Maestre, J. M. et al. (eds.), Actas del IV Congreso Internacional de Humanismo y pervivencia del Mundo Clásico. Homenaje al profesor Juan Gil Fernández, III, Alcañiz, 1163-1190.

Blebel, T. (1584), Rhetoricae artis progymnasmata: Ex Optimis Quibusque Autoribus Methodice conscripta, exemplisque tam sacris, quam philosophicis illustrata, \& ad puerilem instiutionem accommodata, Leipzig. Disponible en: < https://goo.gl/AoUjKk > [16/02/2016].

CERL THESAURUS: accessing the record of Europe's book heritage: $<$ http://thesaurus.cerl. org/record/cnp01333437> [16/02/2016].

Lorich, R. (1598), Aphthonii sophistae Progymnasmata partim a Rodolpho Agricola, partim a Ioanne Maria Catanaeo latinitate donata; cum luculentis \& utilibus eadem sholiis Reinhardi Lorichii Hadamarij, Francoforti. Disponible en: < https://goo.gl/ulUTIX> [16/02/2016].

Lossius, L. (1600), Erotemata Dialecticae et Rhetoricae Philippi Melanthonis et Praeceptionum Erasmi Roterodami, Witerbergae. Disponible en: < https://goo.gl/LRmpL8> [16/02/2016].

Ludovici, G. (1708), Historia rectorum, gymnasiorum scholarumque celebriorum, I, Lanckisius, Biblioteca Estatal de Baviera, pp.283-289.

Melanchthon, Ph. (1537), Elementorum rhetorices libri duo, Argentorati. Disponible en

$<$ https://goo.gl/wpwoMv> [16/02/2016].

Melanchthon, Ph. (1541), Liber Selectarum Declamationum Philippi Melanthonis quas conscripsit, \& partim ipse in schola Vitebergensi recitauit, partim aliis recitandas exhibuit, Argentorati. Disponible en: < https://goo.gl/UECDBM > [16/02/2016].

Rodríguez Herrera, G. (2014), «Etimología y definición en la In Aphthonii Sophistae Progymnasmata Commentario de Buckhardus Harbart», CFC(L) 34.2, 267-281.

Rodríguez Herrera, G. (2016), «Los progymnasmata en los Praeceptionum rhetoricarum libri $V$ et exercitationum libri II de Georg Henisch: fuentes y materiales», Euphrosyne 44, 173-188. 